\title{
Series
}

\section{What history tells us XLI. Ubiquitin and proteolysis}

\author{
MICHEL MORANGE \\ Centre Cavaillès, République des Savoirs: Lettres, Sciences, \\ Philosophie USR 3608, Ecole Normale Supérieure, 29 Rue \\ d'Ulm, 75230, Paris Cedex 05, France \\ (Fax,33-144-323941; Email,morange@biologie.ens)
}

\section{Introduction}

The discovery of the role of ubiquitin in protein degradation may appear simple. In only five years (1978-1982), in a series of articles, Avram Hershko, Aaron Ciechanover and Irwin Rose described the mechanisms by which multiple ubiquitin molecules are linked to a protein by covalent isopeptide linkages in an ATP-dependent way, a first step towards their degradation. They also described the mechanism permitting the recycling of ubiquitin (Ciechanover et al. 1978,1980; Hershko et al. 1979, 1980; Haas et al. 1982; Hershko and Ciechanover 1982). The award to these researchers of the Nobel Prize in Chemistry in 2004 was unanimously praised.

My objective in this contribution is not to describe the details of this highly complex machinery of protein degradation. I will show that this discovery was not as straightforward as it might appear. First, the discovery and the characterization of the functional roles of ubiquitin were the results of a tortuous history. Second, the context in which this discovery was made was complex: the existence and significance of an ATP-dependent degradation of proteins was a hot spot of controversy.

\section{From lymphocyte differentiation and chromatin decondensation to protein degradation}

The discovery of ubiquitin occurred in the field of immunology. After the role of the thymus in lymphocyte maturation had become obvious in the 1960s, the mechanism and in particular the role that polypeptide hormones produced by the thymus might play were extensively scrutinized. In 1973, two of these peptides were described (Scheid et al. 1973). The next year, Gideon Goldstein called these polypeptides thymins (later rebaptized by him thymopoietins to avoid the confusion with the nucleobase) (Goldstein 1974). One of these peptides was shown to have been conserved in evolution from (wrongly) bacteria to higher organisms, and for this reason was called UBIP first, for ubiquitous immunopoietic polypeptide, and then ubiquitin (Goldstein et al. 1975). Ubiquitin was also shown to be an activator of adenylate cyclase, suggesting a mechanism for its role in the differentiation of lymphocytes. Its presence in all tissues of an organism rapidly convinced scientists that ubiquitin had not an active instructive role in the process of differentiation, but rather acted as a trigger on already determined lymphocytes (Scheid et al. 1975). This triggering action did not mean that the role of ubiquitin in lymphocyte differentiation was not significant. It recalled observations made in the 1930s on the Spemann organizer whose action could be mimicked by a series of chemical molecules. More puzzling were later observations showing that ubiquitin had no effect at all on the differentiation of lymphocytes (Low et al. 1979).

In parallel and totally independently, ubiquitin was progressively characterized as a component of chromatin. 1975 saw the report of the presence in chromatin of a minor component called A24, which was later shown to be the result of a tight association between histone $\mathrm{H} 2 \mathrm{a}$ and a non-histone moiety. It was also shown that this non-histone moiety could be linked to histone $\mathrm{H} 2 \mathrm{~b}$. The existence of an isopeptide linkage between the two molecules was demonstrated in 1977 (Goldknopf and Busch 1977). In 1979, it was shown by sequencing that the non-

Keywords. Biochemistry vs. molecular biology; energy dependence; lysosome; nucleosome; proteolysis; ubiquitin 
histone moiety was ubiquitin (Low and Goldstein 1979). A series of studies were rapidly devoted to the functional role that this modification of histone might have, based on precise physical-chemical studies of the modifications of the nucleosome structure that might result from the addition of the nonhistone moiety. Two partially antagonistic models emerged. The rapid disappearance of $\mathrm{A} 24$ (now uH2a) during mitosis suggested that this modification of histones might prevent chromosome condensation (Matsui et al. 1979). Since the non-histone moiety remained present in the nucleus after chromosome condensation, the existence of an amidase able to disrupt the isopeptide linkage was hypothesized. At the beginning of the 1980s, Alexander Varshavsky proposed another function for this modification of histones, based on the development of a new technique that separated nucleosomes harbouring different types of histone modifications and characterized the DNA sequences with which they were associated (Levinger and Varshavsky 1980). A24 was shown to be present in the promoters of actively transcribed genes, suggesting that this modification might be involved in the decondensation of chromatin that occurs during transcription (Levinger and Varshavsky 1982).

It was only in 1980 that ubiquitin entered the domain of protein degradation. Through fractionation of reticulocyte lysates by chromatography, it had been shown by Hershko, Ciechanover and Rose that a thermostable protein factor called APF-1 was covalently linked to proteins as a first step in their degradation. The relation between ubiquitin and APF-1 was made by Michael Urban, a post-doc working close to Rose's laboratory, during a discussion that he had with members of this laboratory (Wilkinson 2005). When he heard about the covalent link between APF-1 and the proteins to be degraded, he remembered the existence of a similar link in the A24 protein. The size of ubiquitin was similar to the size of APF-1, which pushed Wilkinson to initiate a series of experiments that rapidly demonstrated that APF-1 was ubiquitin (Wilkinson et al. 1980). This story is a wonderful example of serendipity as well as of the tortuous ways by which discoveries may occur. But the observations on ubiquitin were not identical: in one case only was ubiquitin involved in protein degradation. It would take many years before it was fully acknowledged that the addition of ubiquitin (as the addition of ubiquitin-like polypeptides) is a signal that can be used for very different purposes: degrading proteins, modifying their functions (as in the case of histones), or addressing proteins to a particular cell compartment as was shown later.

\section{The context of this discovery}

The history and significance of the discovery of the role of ubiquitin in protein degradation cannot be fully understood independently of the historical context in which it occurred; more precisely, of the two different contextual layers that surrounded its birth.

The first layer is present for every discovery. New observations are always interpreted through the perspectives opened by the most recent scientific developments. The importance given to the study of thymic hormones was the consequence of the revolution in immunology made by the discovery of the role of the thymus in the differentiation of lymphocytes, and the description of a second class of lymphocytes, the T lymphocytes. The attribution to these hormones extracted from the thymus of an activating effect on adenylate cyclase is also the result of the impact that the characterization of cyclic AMP as a second messenger had in the 1970s.

Similarly, the focus put on A24 was in direct relation to the characterization in 1974 of the nucleosome, and the new challenges but also opportunities that the description of this structure raised in understanding the structure of chromosomes and the control of transcription (Morange 2013).

The context of the discovery was different for protein degradation. This second context was linked to the general issues raised by the development of molecular biology in the period extending from the mid-1950s to the end of the 1970s.

A first question was the place of lysosomes in proteolysis (De Duve and Wattiaux 1966). Since its discovery by Christian de Duve, there had been accumulating evidence that this organelle could not be the unique and even major pathway of protein degradation in eukaryotes. In particular, it was difficult (though not wholly impossible) to imagine how lysosomes, present in every cell at any time, could be involved in a specific and regulated process of proteolysis.

Behind the search for degradative pathways other than lysosomes was hidden a more central issue: the role of protein degradation in the functional and developmental variations of proteins observed in the cells of higher organisms. The deciphering of the mechanisms controlling gene expression in bacteria - the operon model (1961) - and the huge impact it had, had convinced molecular biologists that variations in the amount of proteins exhibited by the different cell types of higher organisms, or by cells after the addition of, for instance, hormones, were a consequence of changes in the level of gene transcription, more precisely in the rate of initiation of gene transcription.

But most embryologists and physiologists were not convinced. The amount of proteins and enzymes might be controlled at other levels: by a differential stability of messenger RNAs, by a controlled access of these mRNAs to the machinery of translation (as had recently been shown to be the case in early embryos), by protein modifications and, last but not least, by a selective degradation of proteins (Schimke 1969; Schimke and Doyle 1970). By isotopic labelling, Rudolf Schoenheimer had obtained data in the 1940s that demonstrated that proteins were not stable, but in a constant dynamic state (Schoenheimer 1942). In contrast, experiments performed in the 1950s had convinced molecular biologists such as Monod that bacterial proteins were stable. In his Nobel Lecture, Monod presented the observations of Schoenheimer as having generated the mistaken idea that biological macromolecules, and in particular proteins, were 
unstable. For him, this hypothesis had been an obstacle that molecular biologists had to overcome (Monod 1966). The search for a non-lysosomal pathway of proteolysis was therefore supported by the conviction that protein degradation was an essential mechanism of cellular control.

The discovery of the ubiquitin degradation pathway was also at the core of the opposition between biochemists - for whom the study of protein degradation had been a familiar subject since the 1930s - and molecular biologists who had a profound contempt for this field of research and for metabolism in general. For molecular biologists, there was also the memory that biochemists had in the 1940s wrongly supported the idea that protein synthesis was simply the reverse of protein degradation, catalyzed by a multi-protease complex (Bartels 1983).

ATP dependence of the non-lysosomal ubiquitin-dependent proteolytic pathway was not required by thermodynamics, since hydrolysis of polypeptides is an exergonic reaction. But this observation was crucial, not only in showing that the new model had nothing in common with past errors, but also as the sign that this degradation pathway was important and controlled. The experiments of Melvin Simpson published in 1953 were abundantly cited by Hershko, Ciechanover and Rose as the first to have shown on liver slices that protein degradation was an energy-dependent process (Simpson 1953). Maybe the importance attached to this experiment is more a product of hindsight than of interpretation at the time. For Simpson, it was only one among many possible interpretations of his results. In subsequent years, Simpson's efforts were targeted towards the development of an in vitro system of protein synthesis, not proteolysis!

The observation that, once linked to proteins, ubiquitin could be detached by the action of an amidase was compared with the proof-reading mechanisms that had recently been demonstrated in DNA replication and protein synthesis, a way to value the discovery of the ubiquitin pathway through a comparison of the mechanisms involved to those revealed by molecular biologists!

The opposition between molecular biologists and biochemists was also an argument over which organisms should be used as models. For molecular biologists, the mechanisms described in bacteria were also probably operating in higher organisms. For biochemists, mechanisms operating in higher organisms were probably different, and could only be revealed by the study of these organisms. For protein degradation, most biochemists considered that the control of protein and enzyme stability was limited in bacteria, but had acquired a dominant role in higher organisms.

All these debates and oppositions were in the minds of actors and observers of the discovery of the ubiquitindependent proteolytic pathway. It gave this discovery its value, but also explains the reluctance of many to fully acknowledge its importance.

\section{Some significant and partially forgotten contributions}

As in all historical descriptions, some contributions have not received the full attention that they deserve. The first is the contribution of Alfred Goldberg. Not only did he publish in the 1970s two important reviews demonstrating the importance of protein degradation and its dependence on ATP (Goldberg and Dice 1974; Goldberg and John 1976), but he also provided many data in favour of the existence, both in bacteria and in eukaryotes, of ATP-stimulated proteases. But his major contribution was the choice of reticulocyte lysates as a model system to study protein degradation (Etlinger and Goldberg 1977). This extract is very rich in proteolytic activities and devoid of lysosomes, which are lost during the formation of reticulocytes. Hershko, Ciechanover and Rose adopted this system to characterize the ubiquitindependent proteolytic pathway.

The second major contribution was that of Alexander Varshavsky. Interested by the function of $\mathrm{uH} 2 \mathrm{a}$, he seized the opportunity offered by the isolation by a Japanese group of a mutant cell line devoid of A24 in non-permissive conditions (Mita et al. 1980). Varshavsky immediately interpreted this observation by the absence of the enzymatic systems necessary to link ubiquitin to $\mathrm{H} 2 \mathrm{a}$. In collaboration with Ciechanover and Finley he demonstrated that the mutation was in the unique gene coding for the enzyme that activates ubiquitin, the first enzyme in the proteolytic pathway (Finley et al. 1984; Ciechanover et al. 1984). This mutant was essential to confirm definitively the quantitative role of the ubiquitin pathway in proteolysis, in cellular systems different from reticulocytes. It showed that not only did this pathway allow the degradation of abnormal proteins, formed for instance after a heat-shock and more generally cellular stress, but also the proteolysis of normal, short-lived proteins. Observations made with this mutant were the only ones in the following years clearly to support the role of the ubiquitin pathway in the control of major cellular processes.

\section{Conclusions}

A brief description of the discovery of the ubiquitin and ATP-dependent proteolytic pathway fully demonstrates how the value given to experiments and their interpretations are dependent on the context in which they are made. The context first means recently obtained important results, such as the characterization of nucleosomes or the importance of the thymus in the differentiation of lymphocytes. A broader context was the rise of molecular biology and the threat that it represented for biochemists, and in particular those working on proteolysis. But the issue was also the similarities or differences between mechanisms operating in bacteria and in higher organisms, the unity and simplicity, or diversity and 
complexity of the mechanisms controlling development and the functions of organisms. Somehow, these issues were as much philosophical as scientific.

I have ended my description in 1984. Full recognition of the importance of the ubiquitin system was not immediate. Recognition of its role in the degradation of abnormal proteins was rapid, in relation with the molecular characterization of the cellular heat-shock response. But arguments in favour of a normal controlling role of this system, such as its involvement in the degradation of the cyclins involved in the progression in the cell cycle, required more time (Glotzer et al. 1991). Similarly, the characterization of the proteasome, the multi-enzymatic complex that degrades ubiquitinated proteins, required many years. But that is another story!

What probably was the most difficult to accept was the extraordinary and apparently useless complexity of the new pathway, which was correlated with a large waste of energy. The same kind of doubts prevailed when chaperones were described in the same years. Since protein folding was a spontaneous process, what justified the existence of complex and energy-consuming chaperones (Morange 2005)?

What had to be abandoned was nothing less than the idea that organisms function optimally, tailored by the action of natural selection. The limits of natural selection (Gould and Lewontin 1979) and the tinkering action of evolution (Jacob 1977) had not yet fully permeated scientists' minds!

\section{Acknowledgements}

I am indebted to David Marsh for his critical reading of the manuscript.

\section{References}

Bartels D 1983 The multi-enzyme programme of protein synthesis. Its neglect in the history of biochemistry and its current role in biotechnology. Hist. Phil. Life Sci. 5 187-219

Ciechanover A, Hod Y and Hershko A 1978 A heat-stable polypeptide component of an ATP-dependent proteolytic system from reticulocytes. Biochem. Biophys. Res. Comm. 81 $1100-1105$

Ciechanover A, Heller H, Elias S, Haas AL and Hershko A 1980 ATP-dependent conjugation of reticulocyte proteins with the polypeptide required for protein degradation. Proc. Natl. Acad. Sci USA 77 1365-1368

Ciechanover A, Finley D and Varshavsky A 1984 Ubiquitin dependence of selective protein degradation demonstrated in the mammalian cell cycle mutant ts85. Cell 37 57-66

De Duve C and Wattiaux R 1966 Functions of lysosomes. Annu. Rev. Pysiol. 28 435-492
Etlinger JD and Goldberg AL 1977 A soluble ATP-dependent proteolytic system responsible for the degradation of abnormal proteins in reticulocytes. Proc. Natl. Acad. Sci. USA 74 54-58

Finley D, Ciechanover A and Varshavsky A 1984 Thermolability of ubiquitin-activating enzyme from the mammalian cell cycle mutant ts85. Cell 37 43-55

Glotzer M, Murray AW and Kirschner MW 1991 Cyclin is degraded by the ubiquitin pathway. Nature 349 132-138

Goldberg AL and Dice JF 1974 Intracellular protein degradation in mammalian and bacterial cells. Annu. Rev. Biochem. 43 835-869

Goldberg AL and John AC 1976 Intracellular protein degradation in mammalian and bacterial cells: Part 2. Annu. Rev. Biochem. 45 747-804

Goldknopf IL and Busch H 1977 Isopeptide linkage between nonhistone and histone 2A polypeptides of chromosomal conjugateprotein A24. Proc. Natl. Acad. Sci. USA 74 864-868

Goldstein G 1974 Isolation of bovine thymin: a polypeptide hormone of the thymus. Nature. 247 11-14

Goldstein G, Scheid M, Hammerling U, Boyse EA, Schlesinger DH and Niall HD 1975 Isolation of a polypeptide that has lymphocyte-differentiating properties and is probably represented universally in living cells. Proc. Natl. Acad. Sci. USA 72 11-15

Gould SJ and Lewontin R 1979 The spandrels of San Marco and the Panglossian paradigm: a critique of the adaptationist programme. Proc. Roy. Soc. London B. 205 581-598

Haas AL, Warms JVB, Hershko A and Rose IA 1982 Ubiquitinactivating enzyme. J. Biol. Chem. 257 2543-2548

Hershko A, Ciechanover A and Rose IA 1979 Resolution of the ATP-dependent proteolytic system from reticulocytes: a component that interacts with ATP. Proc. Natl. Acad. Sci. USA 76 3107-3110

Hershko A, Ciechanover A, Heller H, Haas AL and Rose I 1980 Proposed role of ATP in protein breakdown: conjugation of proteins with multiple chains of the polypeptide of ATPdependent proteolysis. Proc. Natl. Acad. Sci. USA 77 1783-1786

Hershko A and Ciechanover A 1982 Mechanisms of intracellular protein breakdown. Annu. Rev. Biochem. 51 335-364

Jacob F 1977 Evolution and tinkering. Science 196 1161-1166

Levinger L and Varshavsky A 1980 High-resolution fractionation of nucleosomes: minor particles, "whiskers", and separation of mononucleosomes containing and lacking A24 semihistone. Proc. Natl. Acad. Sci. USA 77 3244-3248

Levinger L and Varshavsky A 1982 Selective arrangement of ubiquitinated and D1 protein-containing nucleosomes within the Drosophila genome. Cell 28 375-385

Low TLK and Goldstein AL 1979 The chemistry and biology of thymosin: II. Amino acid sequence analysis of thymosin $\alpha 1$ and polypeptide $\beta 1$. J. Biol. Chem. 254 987-995

Low TLK, Thurman GB, McAdoo M, McClure J, Rossio JL, et al. 1979 The chemistry and biology of thymosin: I. Isolation, characterization, and biological activities of thymosin $\alpha 1$ and polypeptide $\beta 1$ from calf thymus. J. Biol. Chem. 254 981-986

Matsui S-I, Seon BK and Sandberg AA 1979 Disappearance of a structural chromatin protein A24 in mitosis: implications for 
molecular basis of chromatin condensation. Proc. Natl. Acad. Sci. USA 76 6386-6390

Mita S, Yasuda H, Marunouchi T, Ishiko S and Yamada M 1980 A temperature-sensitive mutant of cultured mouse cells defective in chromosome condensation. Exp. Cell Res. 126 407-416

Monod J 1966 From enzymatic adaptation to allosteric transitions. Science 154 475-483

Morange M 2005 The discovery of chaperone function. J. Biosci. 30 461-464

Morange M 2013 The long and tortuous history of epigenetic marks. J. Biosci. 38 451-454

Scheid MP, Hoffmann MK, Komuro K, Hammerling U, Abbott J, et al. 1973 Differentiation of T cells induced by preparations from thymus and by nonthymic agents. J. Exp. Med. 138 1027-1032

Scheid MP, Goldstein G and Boyse EA 1975 Differentiation of T cells in nude mice. Science 190 1211-1213
Schimke RT 1969 On the roles of synthesis and degradation in regulation of enzyme levels in mammalian tissues. Curr. Top. Cell Reg. 1 77-124, Horecker BC and Stadman BR eds (New York: Academic Press)

Schimke RT and Doyle D 1970 Control of enzyme levels in animal tissues. Annu. Rev. Biochem. 39 929-976

Schoenheimer R 1942 The dynamic state of body constituents (Cambridge: Harvard University Press)

Simpson MV 1953 The release of labeled amino acids from the proteins of rat liver slices. J. Biol. Chem. 201 $143-154$

Wilkinson KD, Urban MR and Haas AL 1980 Ubiquitin is the ATP-dependent proteolysis Factor I of rabbit reticulocytes. J. Biol. Chem. 255 7529-7532

Wilkinson KD 2005 The discovery of ubiquitin-dependent proteolysis. Proc. Natl. Acad. Sci. USA 102 15280-15282 